\title{
The Long Term Effectiveness of Genicular Nerve Blocks for Chronic Knee Pain: A Retrospective Study
}

\author{
Laura Anne Lynem, MD¹, David Lee, MD¹, Brandon Stokey, MD¹, Nguyen Bui, MD', \\ Edgar Martinez, MD², Hossam Ajabnoor, MBBS', Denise Zeller, MSN', and Joseph N. Atallah, MD²
}

Chronic knee pain is a leading cause of chronic disability and its symptoms have a profound impact on the lives of those suffering from it. Current treatment modalities for chronic knee pain include pharmacologic and nonpharmacologic interventions. Genicular nerve blocks are a new therapeutic alternative that have been shown to relieve chronic knee pain and may provide significant relief in patients who are either poor surgical candidates or are unwilling to accept the risks associated with surgery. Genicular nerve blocks may provide long term pain relief for chronic knee pain and radiofrequency ablation (RFA) may not be necessary. This Institutional Review Board (IRB) approved retrospective study conducted at a single center university hospital involved 44 patients aged 43 to 87 who received genicular nerve blocks for chronic knee pain. The primary purpose of this study was to determine if genicular nerve blocks provide effective long term pain relief for patients with chronic knee pain. The numeric rating scale was used to measure the patient's knee pain at baseline, 30,90 , and 120 days post procedure. Patients experienced a mean pain level of 7.43 out of 10 before the genicular nerve block and 4.02 afterward representing an average improvement of $42.6 \%$ in pain relief overall post procedure follow-up. At-test revealed a statistically significant difference between the mean pain score before and after the blocks $(\mathrm{t}=$ 8.534, $P<0.01)$. Overall, the patients experienced significant pain relief of an average of 111.9 days resulting in an average of 42.6 percent pain relief post procedure follow-up. Out of the 44 patients, $15(34.1 \%)$ underwent a RFA after receiving a genicular nerve block procedure to further manage their chronic knee pain symptoms. In conclusion, we have found statistically significant evidence that genicular nerve blocks provide effective long-term pain relief for patients with chronic knee pain. Therefore, for patients who are not surgical candidates, genicular nerve blocks represent a viable alternative treatment for knee pain.

Key words: Genicular nerve blocks, chronic knee pain, genicular nerves, genicular nerve radiofrequency ablation
Chronic knee pain is particularly important due to its high prevalence, especially presenting in younger patients (1). Currently the known treatment modalities for chronic knee pain include pharmacologic and non-

From : 1Department of Anesthesiology, University of Toledo Medical Center, Toledo, OH; 2Division of Pain Medicine, Department of Anesthesiology, University of Toledo Medical Center, Toledo, $\mathrm{OH}$

Author for correspondence: Laura Anne Lynem, MD

Address: Department of Anesthesiology, University of Toledo Medical Center, Mailstop \#1137, Toledo, OH 43614

E-mail: 1al706@gmail.com pharmacologic intervention (2). The current known pharmacologic therapies to treat chronic knee pain include acetaminophen, nonsteroidal anti-inflammatory drugs (NSAIDs), opioids, viscosupplementation, and intraarticular corticosteroid injections. Nonpharmacologic interventions are typically used as adjuncts with the pharmacological therapies, which include such therapies as heat/cold compress, weight loss, exercise, and physical or occupational therapy in an effort to reduce pain and improve functional status (2). Surgical treatments for advanced chronic knee pain may include arthroscopy, osteotomy, and total knee arthroplasty. The latter is typically reserved for 
patients who are unable to perform activities of daily living (ADLs) despite maximal physical therapy (3). Genicular nerve blocks are a new therapeutic alternative that have been shown to relieve chronic knee pain (4). The procedure targets the articular branches of the femoral, obturator, common peroneal, tibial, and saphenous nerves surrounding the knee joint collectively known as the genicular nerves, using fluoroscopic guidance $(5,6)$. Genicular nerve blocks may enhance compliance with physical therapy, potentially delaying total knee arthroplasty. Currently, there are no studies supporting the use of genicular nerve blocks for long-term pain relief for knee pain and, consequently, we seek to further investigate the effectiveness of genicular nerve blocks for the treatment of chronic knee pain.

\section{METHODS}

The purpose of our retrospective study was to assess the clinical efficacy of genicular nerve blocks in providing long term pain relief for patient suffering from chronic knee pain. After obtaining Institutional Review Board (IRB) approval, appropriate protocols were utilized to review electronic medical records at our university institution. The inclusion criteria comprised patients of both genders, at least 18 years of age, with a history of chronic knee pain refractory to conservative management (e.g., physical therapy, oral analgesics, intraarticular joint injections with local anesthetics, or intraarticular injection) who underwent a genicular nerve block procedure between January 2013 and April 2016 (7). The exclusion criteria consisted of patients who do not meet the inclusion criteria. Patients were referred to our pain management center for chronic knee pain if conservative management failed, if they were not surgical candidates secondary to multiple medical comorbidities or if they preferred less invasive management. Each patient underwent a successful unilateral or bilateral genicular nerve block procedure after thorough evaluation confirmed the diagnosis of chronic knee pain. The genicular nerve block procedure begins with the identification of the superior lateral genicular nerve, superior medial genicular nerve, and inferior medial genicular nerve branches under fluoroscopic guidance $(6,8)$. Next, the patient is injected with a combination of $2 \mathrm{~mL}$ of bupivacaine $0.25 \%$ and $20 \mathrm{mg}$ depomedrol at the target site of each genicular nerve. Pre-operative baseline pain characteristics as well as
30,90 , and 120 days post procedure outcomes were measured using the numeric rating scale (NRS) to determine the long-term effectiveness the genicular nerve block. The primary outcomes were the selfreported mean changes in chronic knee pain level baseline to 30,90 , and 120 days and duration of pain relief post procedure per chart review. The secondary outcomes were the number of patients proceeding to genicular nerve radiofrequency ablation (RFA), per chart review. Our statistical analyses were performed at our university institution, using SPSS (IBM Corp. Released 2013. IBM SPSS Statistics for Windows, Version 22.0. Armonk, NY: IBM Corp). A paired sample t-test was performed to assess for statistical significance comparing mean NRS pre-genicular nerve block to post-genicular nerve block at 30, 90, and 120 days, and the average duration between initial pain relief and the reemergence of baseline pain score significance defined with $P<0.01$.

\section{RESULTS}

The retrospective study included a total of 44 patients (men $=9$, women $=35$ ), aged 43 to 87 (mean age 62.7), and an average BMI of 35.4 , suffering from chronic unilateral or bilateral knee pain who underwent unilateral or bilateral genicular (unilateral $=38$, bilateral $=6$ ) nerve block procedures. After IRB approval, each patient's chart was reviewed comparing pre- and post- genicular nerve block NRS using the NRS at baseline and 30,90, and 120 days post procedure. Additionally, the duration of pain relief, number of total genicular nerve blocks, and number of subsequent RFAs were recorded. Our patients reported a mean pain score of 7.43 pre-procedure and a mean pain score of 4.02 overall post procedure follow-up . A t-test revealed a statistically significant difference between the scores before and after the nerve blocks $(t=8.534, P<0.01)$. Overall, the patients experienced significant pain relief of an average of 111.9 days resulting in an average of 42.6 percent pain relief post procedure follow-up. Only 15 (34.1\%) of the 44 patients underwent RFA to further manage their chronic knee pain symptoms after receiving a genicular nerve block.

\section{DISCUSSION}

This is one of the first retrospective studies demonstrating the clinical efficacy of genicular nerve block procedures for the treatment of chronic knee pain. 
Our study found statistically significant evidence that genicular nerve blocks provide effective long-term analgesia for patients suffering from chronic knee pain. However, a genicular nerve block is an invasive procedure and should only be considered when conservative therapy has failed in treating chronic knee pain. Genicular nerve block outcomes are dependent on clinician skill (e.g., ability to identify landmarks correctly). Importantly the superior lateral, superior medial, and inferior medial genicular nerves have a corresponding blood vessel running closely to the surrounding structures that could interfere with the genicular nerve block procedure $(5,10)$. The sensible approach is to precisely target the superior lateral genicular nerve, superior medial genicular nerve, and inferior medial genicular nerves branches, as they course periostealy connecting the femur and tibial shafts to their corresponding epicondyles using fluoroscopic guidance $(5,8)$. Three out of $44(0.68 \%)$ patients were lost to follow-up after the initial 30 days post procedure follow-up and did not return for future genicular nerve block procedures. Unfortunately, 6 out of 44 of the patients (14\%) had little to no pain relief after multiple genicular nerve block procedures. This could possibly be due to anatomical variations of their articular nerves innervating the knee resulting in lack of pain relief post procedure $(5,11)$. Conversely, 10 out of $44(23 \%)$ patients experienced pain relief for 3 months or more after receiving unilateral or bilateral genicular nerve blocks. Patients who are poor surgical candidates or need to delay knee surgery

\section{REFERENCES}

1. Bliddal $\mathrm{H}$, Christensen $\mathrm{R}$. The treatment and prevention of knee osteoarthritis: A tool for clinical decision-making. Expert Opin Pharmacother 2009; 10:1793-1804.

2. Hayami T. Osteoarthritis of the knee joint as a cause of musculoskeletal ambulation disability symptom complex (MADS). Clin Calcium 2008; 18:1574-1580.

3. Zhang W, Moskowitz RW, Nuki G, Abramson S, Altman RD, Arden N, Bierma-Zeinstra S, Brandt KD, Croft P, Doherty M, Dougados M, Hochberg M, Hunter DJ, Kwoh K, Lohmander LS, Tugwell P. OARSI recommendations for the management of hip and knee osteoarthritis, Part II: OARSI evidence-based, expert consensus guidelines. Osteoarthritis Cartilage 2008; 16:137-162.

4. Mathur R. Poster 463 Novel use of genicular nerve block as an alternative treatment option in a patient with chronic bilateral knee pain after left total knee arthroplasty. A case report. PM $R$ 2014; S348.

5. Kennedy JC, Alexander IJ, Hayes KC. Nerve supply of the human knee and its functional importance. Am J Sports Med 1982; 10:329-335. could benefit from long-term genicular nerve block procedures (9). Even patients that do not require total knee arthroplasty can benefit from a genicular never block to treat chronic knee pain. Patients receiving genicular nerve blocks may enhance compliance with physical therapy and increase their range of motion and physical activity (10). According to Le (11), although vascular injuries from genicular RFAs are rare, they carry a significant risk for pseudoaneurysm, arteriovenous fistula, hemarthrosis, and/or osteonecrosis of the patella. Further larger scaled studies will be necessary to validate the long-term effectives of genicular nerve blocks in treating chronic knee pain. Longer follow-up periods in conjunction with larger patient populations from other medical institutions will help elucidate more information on the effectiveness of genicular nerve blocks.

In conclusion, genicular nerve blocks may be a safe and effective procedure to treat chronic knee pain. They are less invasive than RFA or total knee arthroplasty and can be repeated multiple times within a year to provide consistent relief. We have found statistically significant evidence that genicular nerve blocks provide effective long-term pain relief for patients with knee pain, however, longer follow-up periods in conjunction with larger patient populations and further larger scale studies are needed to validate the long-term effectives of genicular nerve blocks in treating chronic knee pain.

6. Hirasawa Y, Okajima S, Ohta M, Tokioka T. Nerve distribution to the human knee joint: Anatomical and immunohistochemical study. Int Orthop 2000; 24:1-4.

7. Zardawi IM, Chan I. Synvisc perisynovitis. Pathology 2001; 33:519-520.

8. Choi WJ, Hwang SJ, Song JG, Leem JG, Kang YU, Park PH, Shin JW. Radiofrequency treatment relieves chronic knee osteoarthritis pain: A double-blind randomized controlled trial. Pain 2011; 152:481-487.

9. Dawson J, Fitzpatrick R, Murray D, Carr A. Questionnaire on the perceptions of patients about total knee replacement. J Bone Joint Surg Br 1998; 80:63-69.

10. Adiguzel E, Uran A, Kesikburun S, Köroğlu Ö, Demir Y, Yaşar $E$. Knee pain relief with genicular nerve blockage in two brain injured patients with heterotopic ossification. Brain Inj 2015; 29:1736-1739.

11. Le PU. Is genicular nerve radiofrequency ablation safe? A literature review and anatomical study. Pain Physician 2016; 19:697705 . 
